\title{
The optics of the low energy FFAG cell of the eRHIC collider using realistic field maps
}

\author{
N. Tsoupas, S. Brooks, A. Jain, F. Meot, \\ G. Mahler, V. Ptitsyn, D. Trbojevic, M. Severance
}

\section{Collider-Accelerator Department Brookhaven National Laboratory Upton, NY 11973}

\section{U.S. Department of Energy Office of Science, Office of Nuclear Physics}

Notice: This document has been authorized by employees of Brookhaven Science Associates, LLC under Contract No. DE-SC0012704 with the U.S. Department of Energy. The United States Government retains a nonexclusive, paid-up, irrevocable, world-wide license to publish or reproduce the published form of this document, or allow others to do so, for United States Government purposes. 


\title{
The Optics of the Low Energy FFAG cell of the eRHIC collider, using realistic field maps*
}

\author{
N. Tsoupas, S. Brooks, A. Jain, F. Méot, G. Mahler, V. Ptitsyn, D. Trbojevic, BNL \\ M. Severance, SBU
}

\begin{abstract}
The proposed electron accelerator of the eRHIC complex [1] will use a $1.32 \mathrm{GeV}$ Energy Recovery Linac (ERL) to accelerate the e-bunches to a top energy of $21.2 \mathrm{GeV}$ before they collide with the hadron bunches. The e-bunches attain the $21.2 \mathrm{GeV}$ energy after passing through the ERL 16 times as they recirculate in two rings which are placed alongside the RHIC hadron accelerator. The two rings [1] are made of periodic cells and each cell is made of one focusing and one defocusing permanent magnet qudrupole. In this paper we present the electromagnetic calculations of the 2D and 3D models of a cell which is comprised of two modified Halbach quadrupoles [4], and the optical properties of the cell.

*Work supported by Brookhaven Science Associate, LLC under Contract No. DE-AC02-98CH10886 with the U.S. Department of Energy.
\end{abstract}

\section{Introduction}

The mission and the design of the electron-ion collider (eRHIC) accelerator to be used in the electron-IonCollisions (EIC) physics program are described in a published report [1]. The eRHIC accelerator, shown schematically in Figure 1 will make use of the existing Relativistic Heavy Ion Collider (RHIC) hadron accelerator which provides various hadronic species having maximum energies ranging from $250 \mathrm{GeV}$ polarized protons to $100 \mathrm{GeV} / \mathrm{u}$ Uranium ions. The electron accelerator of the eRHIC complex will be built alongside the hadron accelerator and will provide electrons with maximum energy of $21.2 \mathrm{GeV}$. The electron accelerator shown as two red rings in Figure 1 will be built alongside the existing hadron accelerator which is shown as a blue ring in Figure 1. Polarized electron bunches will be injected into the ERL at an energy of $12 \mathrm{MeV}$ and will receive an energy increase of $1.32 \mathrm{GeV}$ each time they pass through the ERL which is shown as insert at the right-top of Figure 1. The electron bunches will recirculate in the two electron rings a total of sixteen times to achieve the maximum energy of $21.2 \mathrm{GeV}$. The "low energy" electron ring will recirculate the e-bunches with five energies, 1.32, 2.64, 3.96, 5.29, and $6.61 \mathrm{GeV}$ and the "high energy" ring will recirculate 11 electron bunches with energies ranging from $7.93 \mathrm{GeV}$ to $21.2 \mathrm{GeV}$. Each ring is based on the FFAG principle and consists of FODO cells which are comprised of one focusing and one defocusing quadrupole. One of the proposed designs of the FODOquadrupoles is a Halbach type permanent magnet quadruple which is modified to avoid the interaction of the permanent magnet material with the synchrotron radiation emitted by the circulating electron bunches. This paper will present the electromagnetic design of the cell's quadrupoles including magnetic interference and the optical properties of the cell using the field map derived from the electromagnetic study of the cell. The beam optics will be compared to that derived using ideal hard edge quadrupoles which do not interfere with each other. 


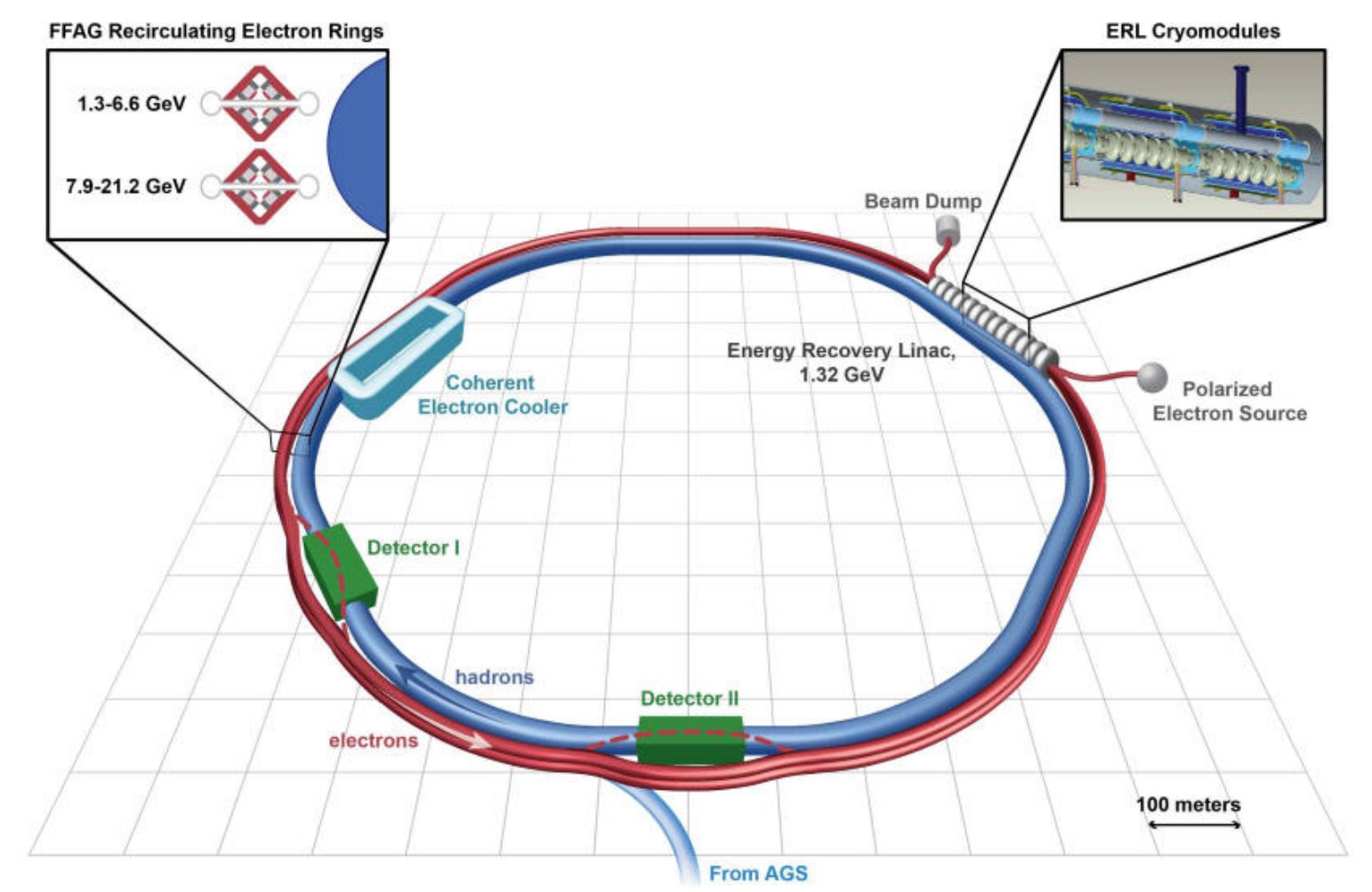

Figure 1. Schematic diagram of the eRHIC accelerator. The electron accelerator (red rings) will be built alongside the existing hadron accelerator (blue ring). Polarized electron bunches will be injected at $12 \mathrm{MeV}$ and will receive $1.32 \mathrm{GeV}$ of energy each time they pass through the ERL whose cutout is shown on the top right insert. The electron bunches recirculate in the two electron rings sixteen times to achieve the maximum energy of $21.2 \mathrm{GeV}$. A cross section of the cell quadrupoles of the electron arcs is show in the top left insert of the figure.

\section{The FFAG cell}

One of the possible cell designs to be used for the low energy ring of the electron accelerator is shown in the schematic diagram of Figure 2. The FODO cell is $168 \mathrm{~cm}$ long and consists of a $48 \mathrm{~cm}$ long focusing and a $44 \mathrm{~cm}$ long defocusing quadrupole. The quadrupoles with aperture of radius $2 \mathrm{~cm}$ are separated longitudinally by $6 \mathrm{~cm}$ and their optical axes are separated transversely by $5.4 \mathrm{~mm}$. The low energy ring consists of 6 arcs with 212 cells per arc, therefore each low energy cell and bends the electron bunches by $4.9396 \mathrm{mrad}\left(0.2830^{\circ}\right)$. The remarkable property of the FFAG cell is indicated in Figure 2 which shows the trajectories of the electron bunches in the large energy range from $1.334 \mathrm{GeV}$ to $6.622 \mathrm{GeV}$ to be contained within a small transverse space of $16.9 \mathrm{~mm}$. Figure 2 shows also the trajectories of the electron bunches in the energy range from $1.332 \mathrm{GeV}$ to $6.622 \mathrm{GeV}$ as calculated by the PTC code [2] which assumes that the quadrupoles are pure with no fringe field (hard edge) or higher order multipoles. 


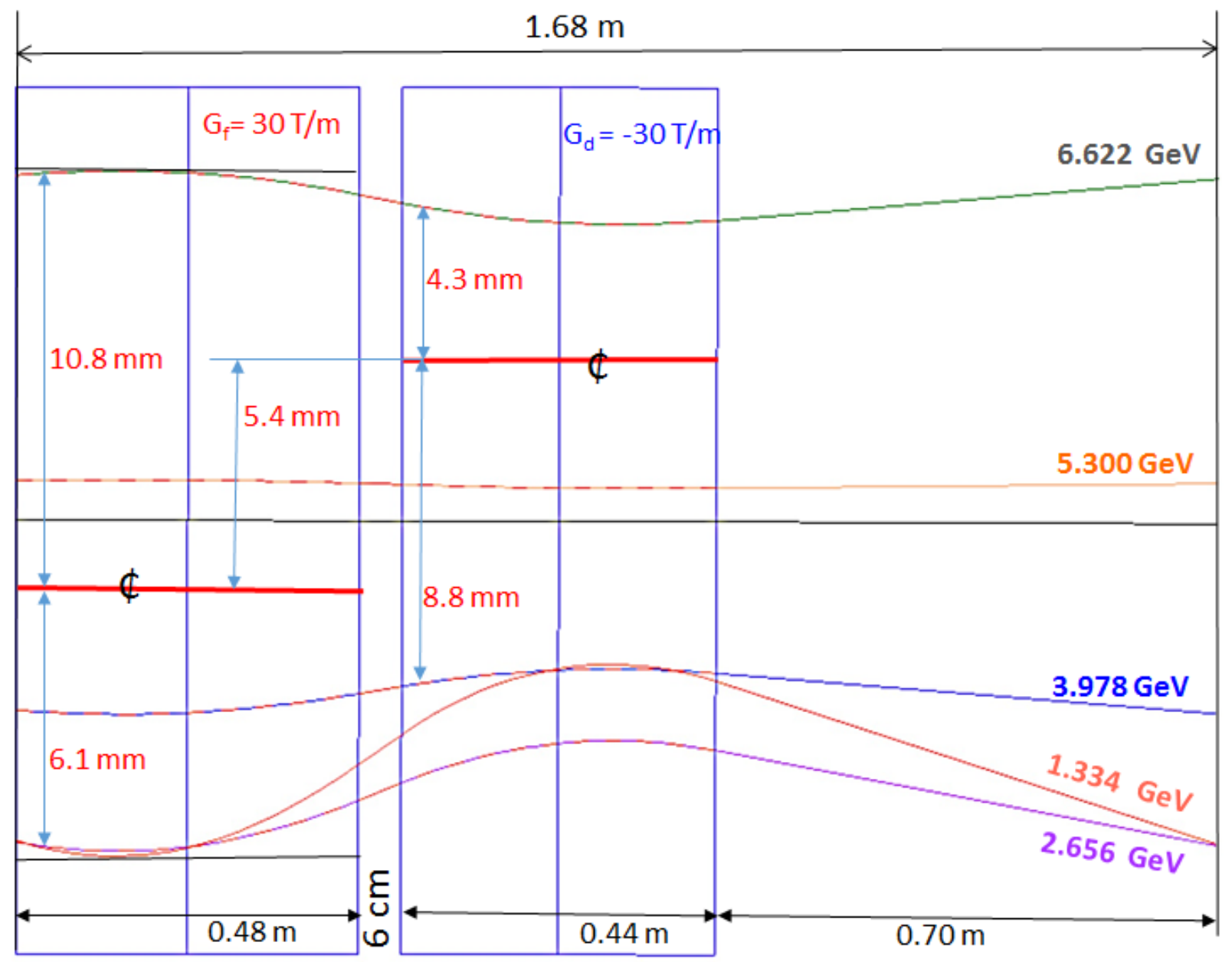

Figure 2. Schematic diagram of the $1.68 \mathrm{~m}$ long low energy FFAG cell. The quadrupoles which have a gradient of $30 \mathrm{~T} / \mathrm{m}$ and $-30 \mathrm{~T} / \mathrm{m}$ respectively are separated in the longitudinal direction by $6 \mathrm{~cm}$ and their symmetry axes (thick red lines) are displaced transversely by $5.4 \mathrm{~mm}$. Each of the trajectories in the energy range 1.334 to $6.622 \mathrm{GeV}$ of the five electron bunches are bent by the quadrupoles by $4.9396 \mathrm{mrad}\left(0.2830^{\circ}\right)$ and are plotted on this Figure. The low energy ring consists of 6 arcs with 212 cells in each arc.

\section{Electromagnetic design of the cell's quadrupoles}

The PTC code calculations for the optics of the FFAG cell assumes that the quadrupoles have no fringe field (hard edge) and are pure with no magnetic multipoles higher than quadrupole. Moreover it assumes that there is no interference between the two quadrupoles in the cell. To calculate the optical properties of the cell with realistic magnetic fields we use the OPERA computer code [3] which provides 3D field maps of the cell's quadrupoles. These calculations provide the fringe field generated by the quadrupoles and the interference of the quadrupoles in a cell and also the interference of the quadrupoles from the neighboring cells. The four fold symmetry of each quadrupole is kept in the design therefore the first allowed magnetic multipoles of the quadrupole is the 12-pole. As we mentioned earlier each cell bends the electron bunches by 4.9396 mrad therefore the longitudinal axis of each cell is rotated by $4.9396 \mathrm{mrad}$ 
about the vertical axis. Figure 3 is a schematic diagram of three consecutive cells which were used in the OPERA code to model the effect of the interference among the cells. The red lines indicate the symmetry axes of the quadrupoles which are separated by $5.4 \mathrm{~mm}$. Each cell is displaced radially by $5.5 \mathrm{~mm}$ and rotated by an angle $\theta=4.9396$ mrad about the vertical axis with respect to the previous cell.

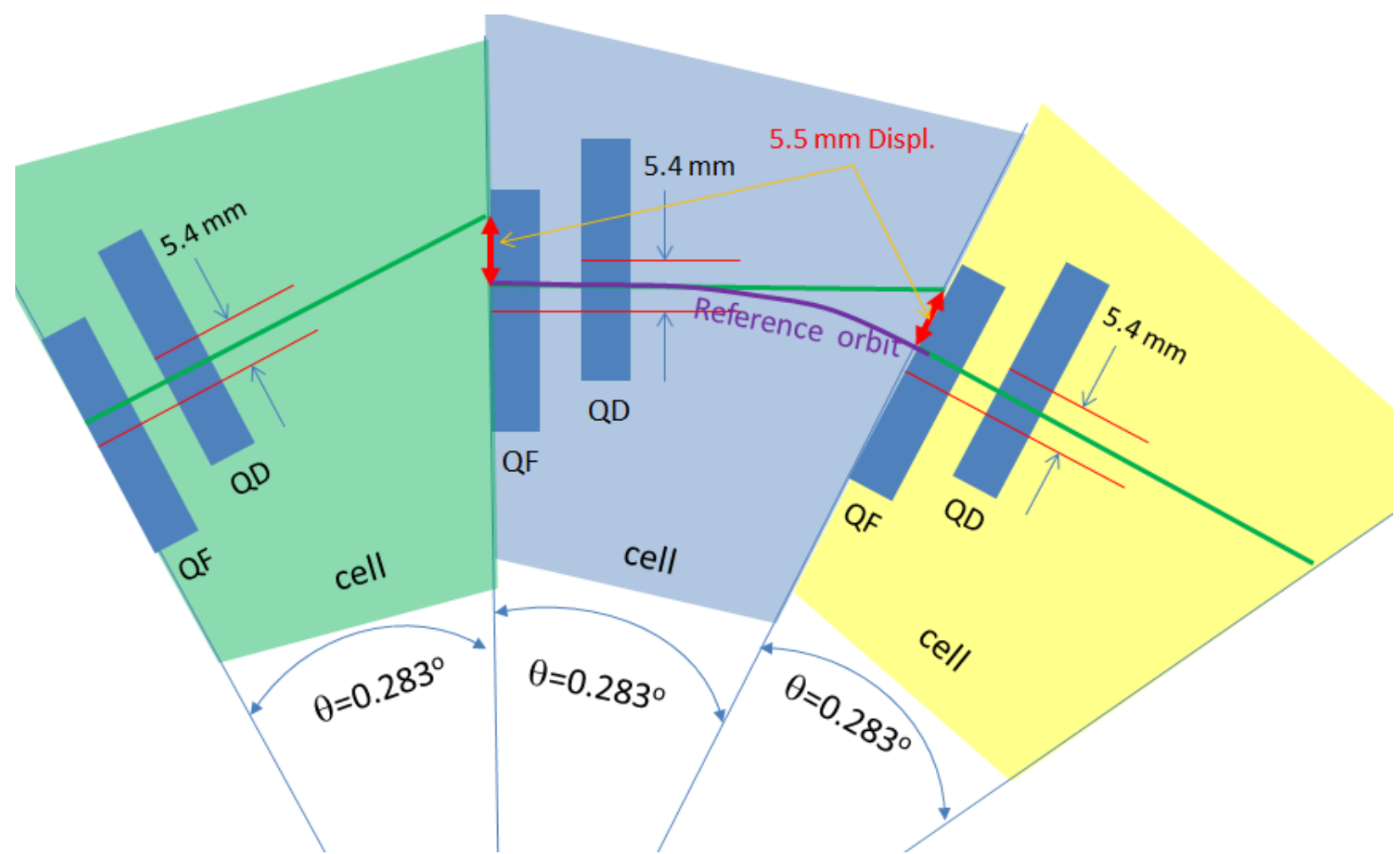

Figure 3. A schematic diagram of three consecutive FFAG cells. Each cell is displaced radially by $5.5 \mathrm{~mm}$ and rotated by an angle $\theta=4.9396 \mathrm{mrad}\left(0.283^{\circ}\right)$ with respect to the previous cell. The red lines indicate the longitudinal symmetry axes of the quadrupoles. The reference orbit for the center cell is also shown. All six quadrupoles were modeled in the 3D OPERA code to take into account the effect of the interference among the quadrupoles of the three cells.

The electromagnetic calculations were performed in two steps, first the 2D OPERA calculations which help define the cross section of the quadrupole and minimize the 12-pole multipole, and second the 3D OPERA calculations which take into account the interference among the quadrupoles and provide the 3D field maps to be utilized in the beam optics calculations.

The 2D Design of the Quadrupole

Two 2D models of the modified Halbach type permanent magnet quadrupoles are shown in Figure 4 and correspond to the design of a Halbach quadrupole having 16 wedges. The modification from the Halbach design consists of removing the permanent magnet wedges from the $0^{\circ}$ and $180^{\circ}$ azimuthal locations to avoid damage from the synchrotron radiation. To keep the four-fold symmetry of the design we also remove the wedges from the $90^{\circ}$ and $270^{\circ}$. The yellow lines drawn on top of the two cross sections of the quadrupoles are the equipotential lines of the vector potential. 

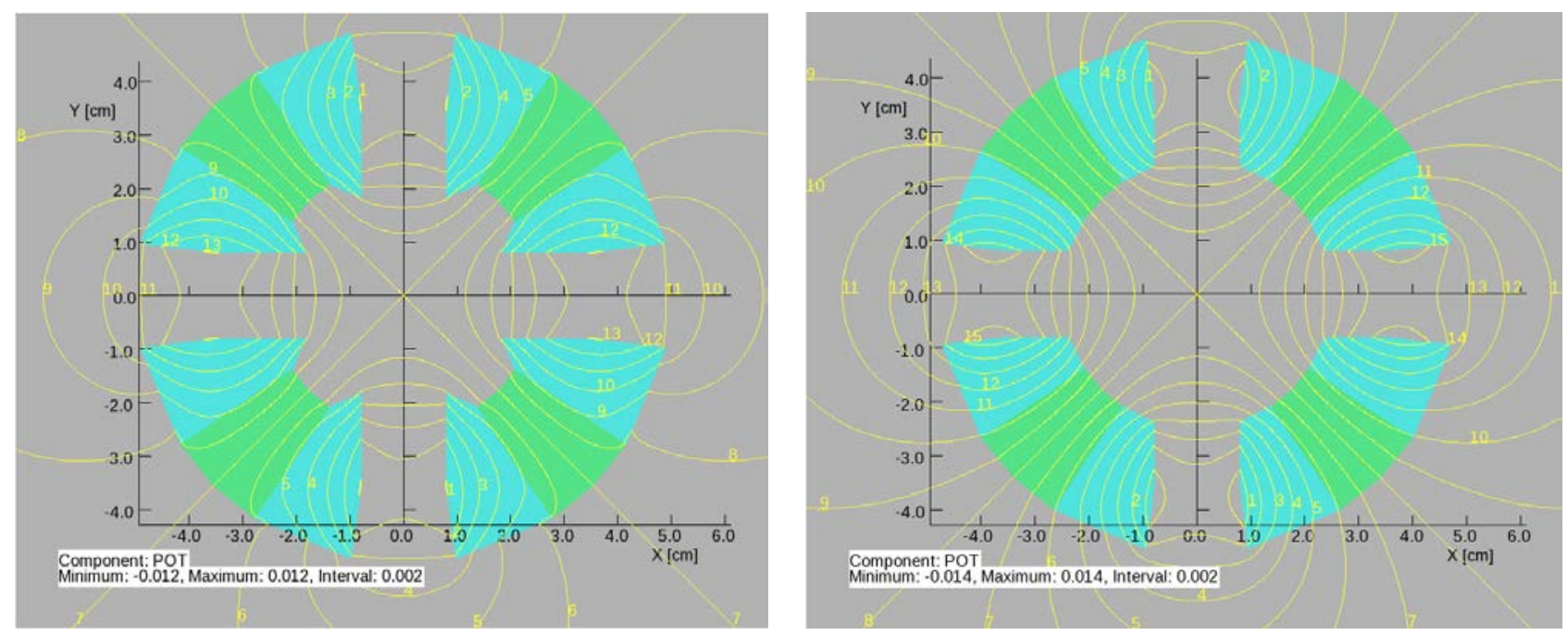

Figure 4. Two 2D models of the modified Halbach quadrupole which provide a Gradient of $30 \mathrm{~T} / \mathrm{m}$. The wedges on the left model are made of SmCo permanent magnet material and the wedges of the right quadrupole are made of $\mathrm{NdFeB}$. Note that both quadrupoles have the wedges at $0^{\circ}, 90^{\circ}, 180^{\circ}$, and $270^{\circ}$, removed to prevent their interaction with the synchrotron radiation and maintain the four-fold symmetry which keeps at zero all multipoles except the allowed ones. The yellow lines drawn on top of the two cross sections of the quadrupoles are the equipotentials of the vector potential.

The 2D design determines the minimum inner radius (aperture) of the quadrupole which will allow for a vacuum chamber and possible corrector coils which will provide the required gradient. Subsequently the first allowed 12-pole multipole is optimized to zero by modifying the shape of the inner sides of the wedges (left picture of Figure 4) and also by changing the magnetization direction of the wedges next to the $45^{\circ}$ and $135^{\circ}$ wedges. The direction of the easy axis of the wedges along the $45^{\circ}$ and $135^{\circ}$ directions are kept as that dictated by the Halbach design. The permanent magnet material of the model shown on the left picture of Figure 4 is SmCo-R26HS and that on the right picture is of NdFeB-N45. The BH curves are shown in Figure 5. Both 2D-models shown in Figure 4 provide a gradient of $30 \mathrm{~T} / \mathrm{m}$ with all magnetic multipoles up to the first allowed 12-pole-multipole included, being zero within a radius of $12 \mathrm{~mm}$. This is accomplished by the four fold symmetry of the model and by changing the direction of the easy axis of each wedge in a symmetric manner with respect to the $45^{\circ}$ and $135^{\circ}$ directions. Table 1 shows the direction of the easy axis of the wedges for the Halbach-type quadrupole ( $2^{\text {nd }}$ row) and the two modified Halbach designs ( $3^{\text {rd }}$ and $4^{\text {th }}$ rows) shown in Figure 3. The shape of some of the wedges of the design shown on the left picture of Figure 4 helps keep the outer radius of the quadrupole less than $5 \mathrm{~cm}$ and also minimize the 12-pole multipole. The design shown on the right picture of Figure 4 avoids this peculiar shape of the wedges of the picture on the left because the remnant magnetization of the NdFeBN45 as shown in Figure 5 is larger than that of the SmCo-R26HS material. Row 1 of Table 1 is the average azimuthal angle of the wedges shown in Figure 4. Rows 2, 3, 4 correspond to the direction of the easy axis of the wedges. The easy axis direction shown in the $2^{\text {nd }}$ row corresponds to the Halbach design, and the directions of the easy axes for the modified designs of the quadrupoles shown in left and right pictures of Figure 4 are shown in the $3^{\text {rd }}$ and $4^{\text {th }}$ rows. 


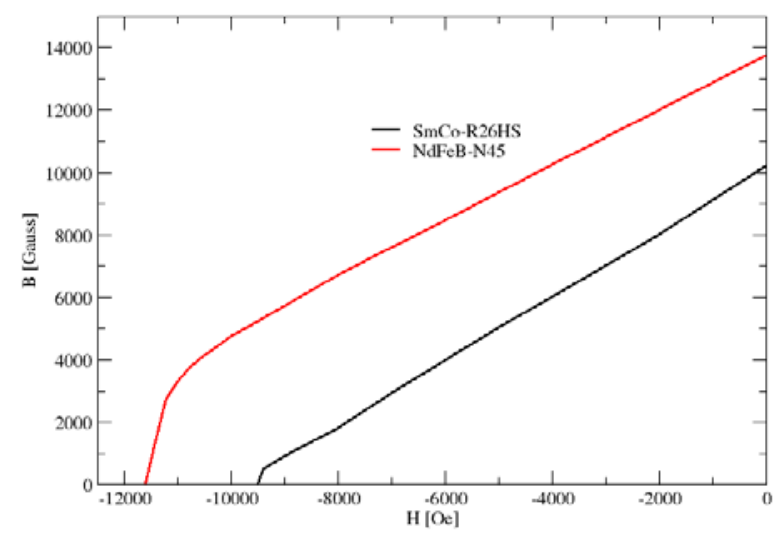

Figure 5. The BH curves of two permanent magnet materials which were used in the magnetic field calculations. The SmCo-R26HS or NdFeB-N45 materials are used as materials for each of the two models shown in Figure 4.

Table 1 . The direction of the easy axis for the wedges a) of an ideal Halbach quad ( $2^{\text {nd }}$ row) b) of a quadrupole shown in the left picture of Figure 4 ( $3^{\text {rd }}$ row) c) of a quadrupole shown in the right picture of Figure $4\left(4^{\text {th }}\right.$ row $)$. The easy axis direction of the quadrupoles in the $3^{\text {rd }}$ and $4^{\text {th }}$ rows is chosen such as to make the 12-pole equal to zero.

\begin{tabular}{|l|l|l|l|l|l|l|l|l|l|l|l|l|}
\hline $\begin{array}{l}\text { Wedge } \\
\text { Angle }\end{array}$ & $0^{\circ}$ & $22.5^{0}$ & $45^{\circ}$ & $67.5^{\circ}$ & $90^{\circ}$ & $112.5^{\circ}$ & $135^{\circ}$ & $157.5^{\circ}$ & $180^{\circ}$ & $202.5^{\circ}$ & $225^{\circ}$ & $247.5^{0}$ \\
\hline Halbach & $90^{\circ}$ & $157.5^{\circ}$ & $225^{\circ}$ & $292.5^{\circ}$ & $0^{\circ}$ & $67.5^{\circ}$ & $135^{\circ}$ & $202.5^{\circ}$ & $270^{\circ}$ & $337.5^{\circ}$ & $45^{\circ}$ & $112.5^{\circ}$ \\
\hline SmCo & NA & $182.5^{\circ}$ & $225^{\circ}$ & $267.5^{\circ}$ & NA & $92.5^{\circ}$ & $135^{\circ}$ & $177.5^{\circ}$ & NA & $362.5^{\circ}$ & $45^{\circ}$ & $87.5^{\circ}$ \\
\hline NdFeB & NA & $204.5^{\circ}$ & $225^{\circ}$ & 245.5 & NA & $114.5^{\circ}$ & $135^{\circ}$ & 155.5 & NA & $384.5^{\circ}$ & $45^{\circ}$ & $65.5^{\circ}$ \\
\hline
\end{tabular}

\begin{tabular}{|l|l|l|l|l|}
\hline $\begin{array}{l}\text { Wedge } \\
\text { Angle }\end{array}$ & $270^{\circ}$ & 292.5 & $315^{\circ}$ & $337.5^{\circ}$ \\
\hline Halbach & $180^{\circ}$ & $247.5^{\circ}$ & $315^{\circ}$ & $22.5^{\circ}$ \\
\hline SmCo & NA & $272.5^{\circ}$ & $315^{\circ}$ & $357.5^{\circ}$ \\
\hline NdFeB & NA & $294.5^{\circ}$ & $315^{\circ}$ & $335.5^{\circ}$ \\
\hline
\end{tabular}

Figure 6 is a colored-zones plot of the B-field-modulus over the cross section of the magnet shown in the left picture of Figure 4. This plot shows that the B-field in most of the permanent magnet material is above 0.26 Tesla. This value is higher than the value of the B-fied which corresponds to the "knee" of the SmCo-R26HS BH curve shown in Figure 5 therefore any variations of the material's temperature will not affect its magnetic properties when the temperature is restored. 


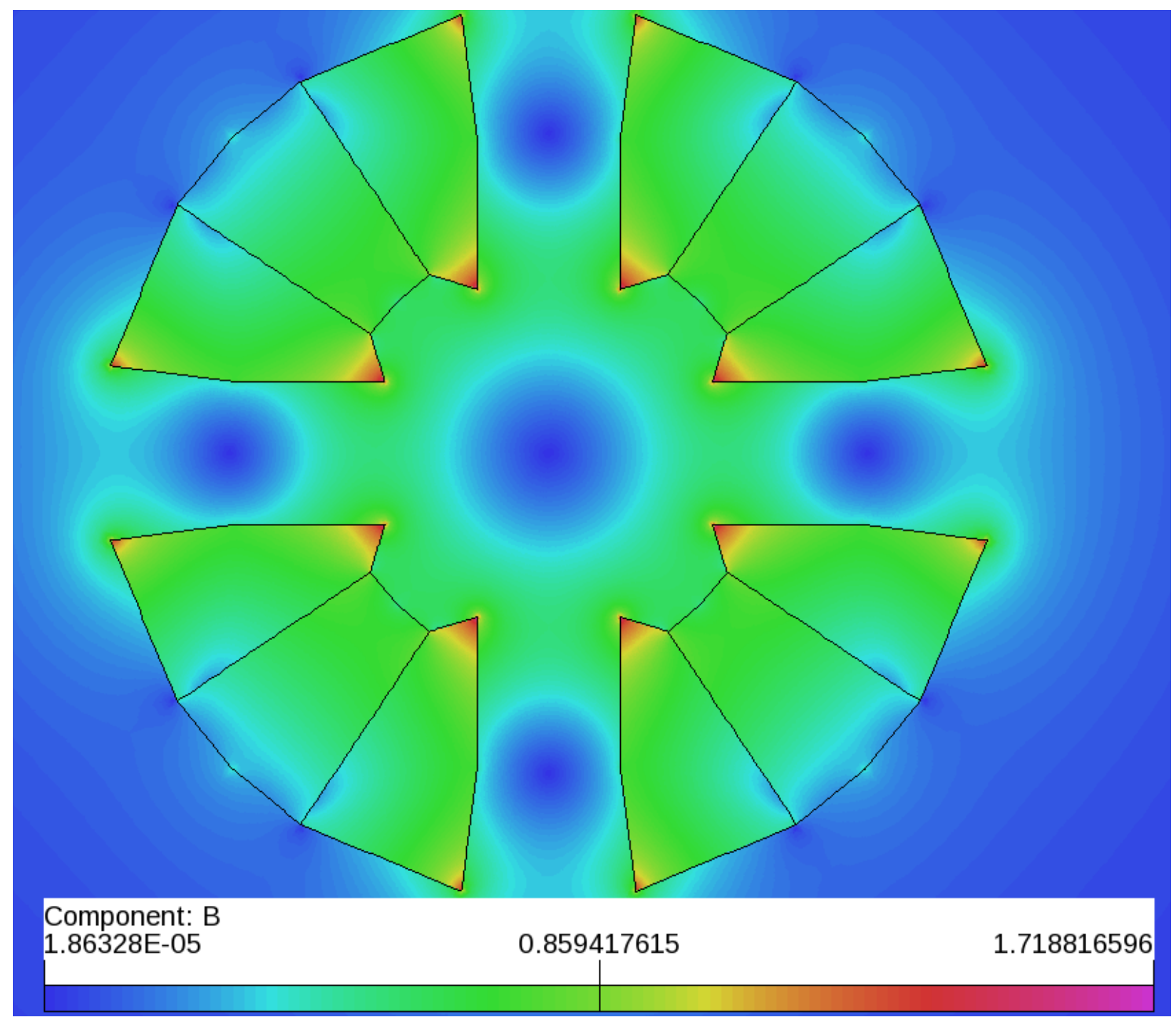

Figure 6. The B-field-modulus over the area of the SmCo-R26HS permanent magnet material of the magnet shown in the left picture of Figure 4. Most of the magnet's material is in B-field larger than 0.26 Tesla.

\section{D Design}

The 2D magnetic design discussed in the previous subsection provides a quick way to set the required gradient of the quadrupole and also minimize the first allowed multipole (12-pole) of the quadrupole. The 3D magnetic design provides accurate information on the field generated by the quadrupoles of the cell, including fringe fields and the interference with the neighboring quadrupole in the same cell as well as in the neighboring cells. Figure 3 is a schematic diagram of the 3 cells which are modeled using the TOSCA module of the OPERA code. The modeling of the 3 cells is the minimum number of cells required to provide accurate information of the magnetic field map of a single cell. The left picture in Figure 7 is an isometric view of the two quadrupoles of one of the three cells used in the OPERA generated model. The magnetization direction of the easy axis of the wedges shown in Figure 7 (left) is identical to that of the 
wedges used in the $2 \mathrm{D}$ modeling, and is shown in the $3^{\text {rd }}$ row of Table 1 . The strength of the multipoles at the center-point of each quadrupole as calculated in the 3D model (for $\mathrm{R}=1 \mathrm{~cm}$ ) are identical to the strengths as calculated by the 2D model therefore no new further optimization was required for the 3D design. This agreement between the 2D and 3D models indicates that there is only superposition of the fields generated by the quadrupoles and the field of a quadrupole is not distorted by the presence of the other. This is due to the nature of the $\mathrm{BH}$ curve (see Figure 5 ) which yields permeability $\mu=\mathrm{B} / \mathrm{H} \approx 1.0$. The TOSCA solver of the OPERA code provides the components of the magnetic field at any point within the volume of the cell. The picture on the right of Figure 7 is an isometric plot of the vertical component of the field $\left(B_{y}\right)$ on a rectangular patch on the median plane $(y=0)$ and centered half way between the two quads of a cell. The patch extends $10 \mathrm{~cm}$ inside each quad (z-direction) and its extent in the $\mathrm{x}$ and $\mathrm{y}$ directions is from $x / y= \pm 2 \mathrm{~cm}$. The components of the magnetic fields become available to the computer code "zgoubi" [5] as a field map on a rectangular grid and are used to calculate the optical properties of the cell. In particular the field map extends on a rectangular grid $168 \mathrm{~cm}$ long along the length of the cell in the z-direction and from $\pm 3 \mathrm{~cm}$ in the $\mathrm{x}$ and $\mathrm{y}$ transverse directions. The grid-step-size is $2 \mathrm{~mm}$. In the next section we study the beam optics of the cell in two ways, one when the cell consists of ideal quadrupoles with no fringe fields and no interaction with each other, and the other using the field maps of a realistic cell as obtained from the 3D TOSCA calculations.
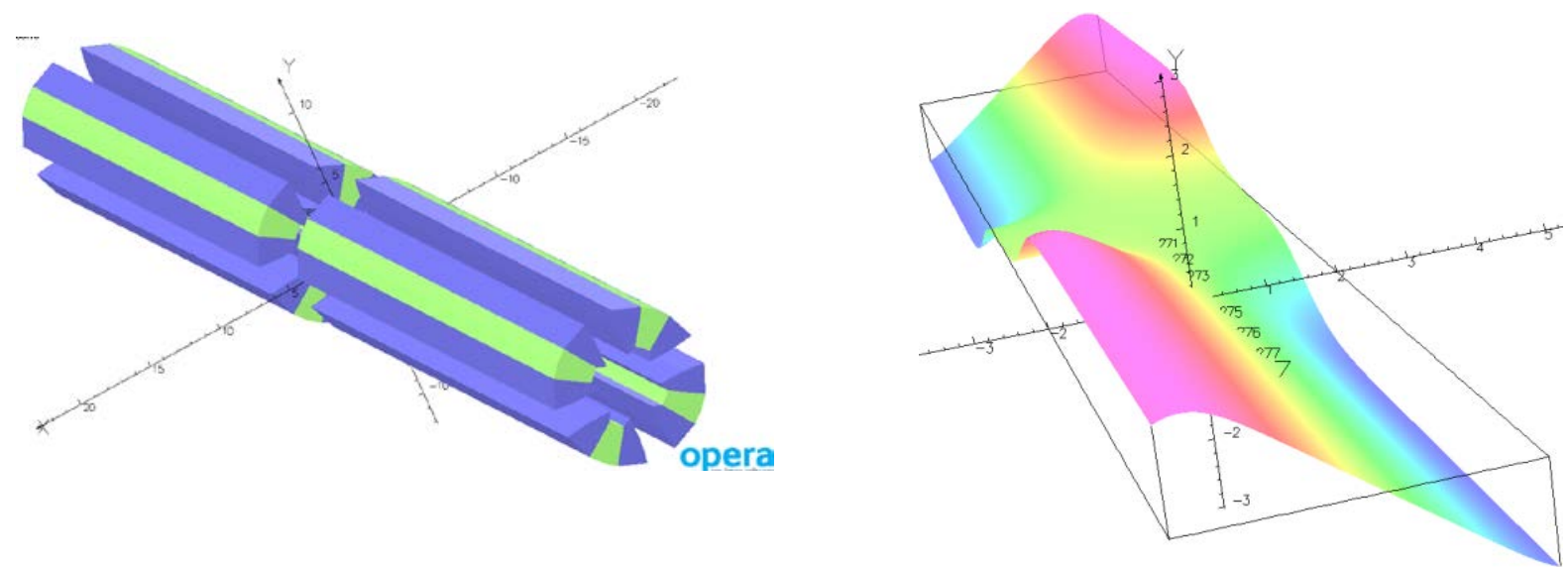

Figure 7. Left: An isometric plot of the two modified Halbach quadrupoles of a cell. Right: The ycomponent $\left(\mathrm{B}_{\mathrm{y}}\right)$ of the B-field over the median plane in the fringe field region of the two quads of the cell. The separation distance between the two quads is $6 \mathrm{~cm}$ and the rectangular patch over which the field is plotted extends from $\mathrm{x}=-2 \mathrm{~cm}$ to $\mathrm{x}=+2 \mathrm{~cm}$ in the $\mathrm{x}$-direction and $10 \mathrm{~cm}$ inside each of the quadrupoles.

\section{Magnetic measurements of a single quadrupole}

To ascertain the agreement of the calculated field maps with the experiment, we performed magnetic field measurements on a single quadrupole which was assembled with wedges having the same cross section as the wedges on the left picture of Figure 4. A picture of the assembled magnet is shown in Figure 8. The housing of the permanent magnet wedges was made with a 3D-printer and the wedges were forced into the openings of the housing. The length of each of the wedges is $6 \mathrm{~cm}$ and the material is SmCo-R26HS with BH curve shown in Figure 5. The direction of the easy axis of the wedges is shown in Table 2. 


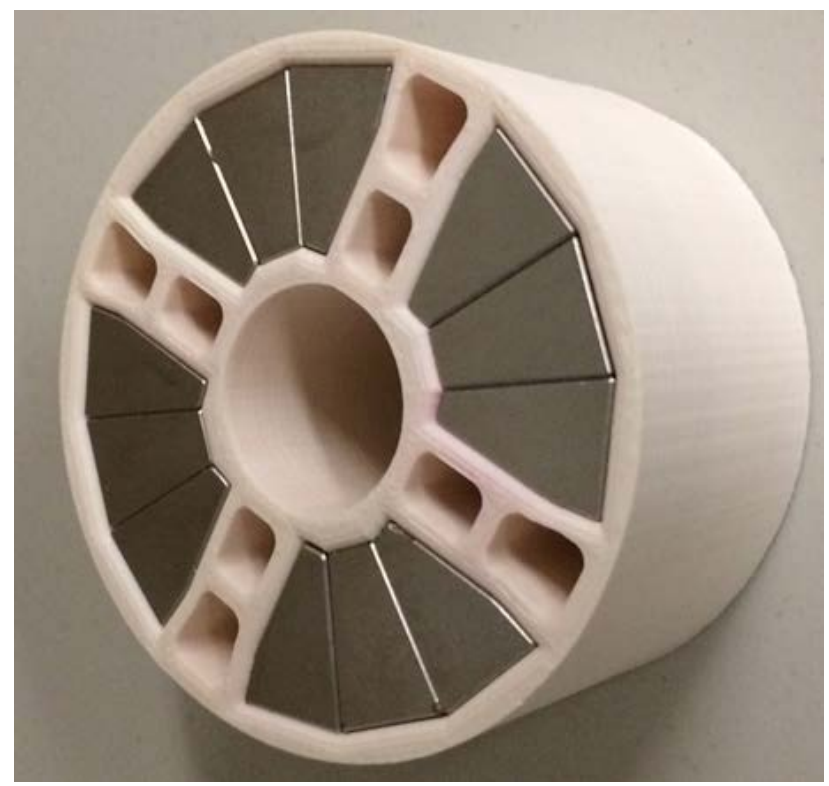

Figure 8. A picture of the actual modified Halbach quadrupole. The housing of the permanent magnet wedges is made by a $3 \mathrm{D}$ printer.

Table 2. The direction of the easy axis for the wedges shown in Figure 7 appear in the $2^{\text {nd }}$ row.

\begin{tabular}{|l|l|l|l|l|l|l|l|l|l|l|l|l|}
\hline $\begin{array}{l}\text { Wedge } \\
\text { Angle }\end{array}$ & $0^{\circ}$ & $22.5^{0}$ & $45^{\circ}$ & $67.5^{\circ}$ & $90^{\circ}$ & $112.5^{\circ}$ & $135^{\circ}$ & $157.5^{\circ}$ & $180^{\circ}$ & $202.5^{\circ}$ & $225^{\circ}$ & $247.5^{\circ}$ \\
\hline SmCo & NA & $157.5^{\circ}$ & $225^{\circ}$ & $292.5^{\circ}$ & NA & $67.5^{\circ}$ & $135^{\circ}$ & $202.5^{\circ}$ & NA & $337.5^{\circ}$ & $45^{\circ}$ & $112.5^{\circ}$ \\
\hline
\end{tabular}

\begin{tabular}{|l|l|l|l|l|}
\hline $\begin{array}{l}\text { Wedge } \\
\text { Angle }\end{array}$ & $270^{\circ}$ & 292.5 & $315^{\circ}$ & $337.5^{\circ}$ \\
\hline SmCo & NA & $247.5^{\circ}$ & $315^{\circ}$ & $22.5^{\circ}$ \\
\hline
\end{tabular}

The measured and calculated integrated quadrupole and 12-pole multipoles at a radius of $\mathrm{R}=1 \mathrm{~cm}$, for the model shown in Figure 8, are shown in $2^{\text {nd }}$ and $4^{\text {th }}$ columns respectively of Table 3 . The $3^{\text {rd }}$ and $5^{\text {th }}$ columns in Table 3 show the difference of the integrated quadrupole and 12-pole multipoles relative to the measured integrated quadrupole.

Table 3. The measured and calculated integrated quadrupole and 12-pole multipoles at a radius of $\mathrm{R}=1$ $\mathrm{cm}$, for the model shown in Figure 8, are shown in $2^{\text {nd }}$ and $4^{\text {th }}$ columns respectively. The $3^{\text {rd }}$ and $5^{\text {th }}$ columns show the difference of the integrated quadrupole and 12-pole relative to the measured integrated quadrupole.

\begin{tabular}{|c|c|c|c|c|}
\hline & $\begin{array}{c}\text { Int_Quad } \\
{[\text { Gauss }]}\end{array}$ & Q_Diff/Q & $\begin{array}{c}\text { Int_12pole } \\
{[\text { Gauss.cm }}\end{array}$ & $\begin{array}{c}\text { 12p_diff } / \mathbf{Q}_{\text {meas }} \\
{\left[\mathrm{cm}^{-4}\right]}\end{array}$ \\
\hline $\begin{array}{c}\text { SmCo R26HS } \\
\text { Temp.=20 } \\
\text { Calculations }\end{array}$ & 18730.5 & & 337.4 & \\
\hline $\begin{array}{c}\text { Magnet\#1 } \\
\text { Measurement }\end{array}$ & 18650.0 & $4.3 \times 10^{-3}$ & 350.6 & $7 \times 10^{-4}$ \\
\hline
\end{tabular}




\section{Optical properties of the cell}

The low energy ring consists of six arcs with each arc containing 212 cells, and recirculates energy bunches with five energies ranging from 1.3 to $6.6 \mathrm{GeV}$. The "beam optics of the cell” is the study of the following properties of the cell:

a) The "closed orbit" of particles within the energy range 1.3 to $6.6 \mathrm{GeV}$. This study will prove that the magnetic field map of a single cell allows the formation of closed orbits within this assumed energy range.

b) The horizontal and vertical tunes $\mathrm{Q}_{\mathrm{x}}$ and $\mathrm{Q}_{\mathrm{y}}$ and of the chromaticities $\xi_{\mathrm{x}}$, $\xi_{\mathrm{y}}$, which correspond to the closed orbits calculated in part (a) above. This study provides information on the stability of the closed orbits.

c) The maximum beam emittances $\varepsilon_{x}$, and $\varepsilon_{y}$, that corresponds to a closed orbit of a bunch with given energy. Starting with each closed orbit as a reference orbit of a beam bunch we calculate the maximum phase space ellipses $\left(\mathrm{x}, \mathrm{x}^{\prime}\right)$ and $\left(\mathrm{y}, \mathrm{y}^{\prime}\right)$ which are transported along the low energy ring.

d) The dynamic aperture for each closed orbit. Starting with each closed orbit as a reference orbit of a beam bunch we calculate the maximum values of the particle coordinates $(x, y)$ which are transported in the low energy ring.

e) The beta functions of the cell.

The above study is performed for two cases; one corresponding to ideal quadrupoles of the cell with no fringe fields and no interaction with each other, and the other with real quadrupoles having fields as calculated by the OPERA computer code which takes into account the interference of the two quads in a cell as well as the interference of the quadrupoles in neighboring cells. In both cases the zgoubi code was used to calculate the optical properties of the cell.

\section{Beam Optics of the cell using OPERA computed field maps}

In this section we present the results of the beam optics calculations by completing the tasks (a) to (e) above. The calculations were performed by the "zgoubi" computer code [5] that made use of the field map of a cell as calculated by the OPERA computer code.

\section{The closed orbit trajectories}

To obtain the closed-orbit trajectories of the bunches as they traverse a cell we used 1272 cells which comprise the low energy ring. The "zgoubi" computer code raytraced particles with a given energy in the 3D field maps of the cells and provided a closed orbit for each energy. Figure 9 shows 44 closed orbits on the median plane in a cell in the energy range from 1.334 to $6.6 \mathrm{GeV}$. This figure demonstrates the remarkable property of the FFAG concept which allows a large energy range of particles to be accommodated in a small transverse distance from -1.5 to $1.0 \mathrm{~cm}$. The coordinate system which is used by the computer code "zgoubi” is: $y$-radial, $x$-along beam, and z-vertical. The yellow shaded regions in Figure 9 correspond to the location of the focusing and defocusing quadrupoles in the cell. 


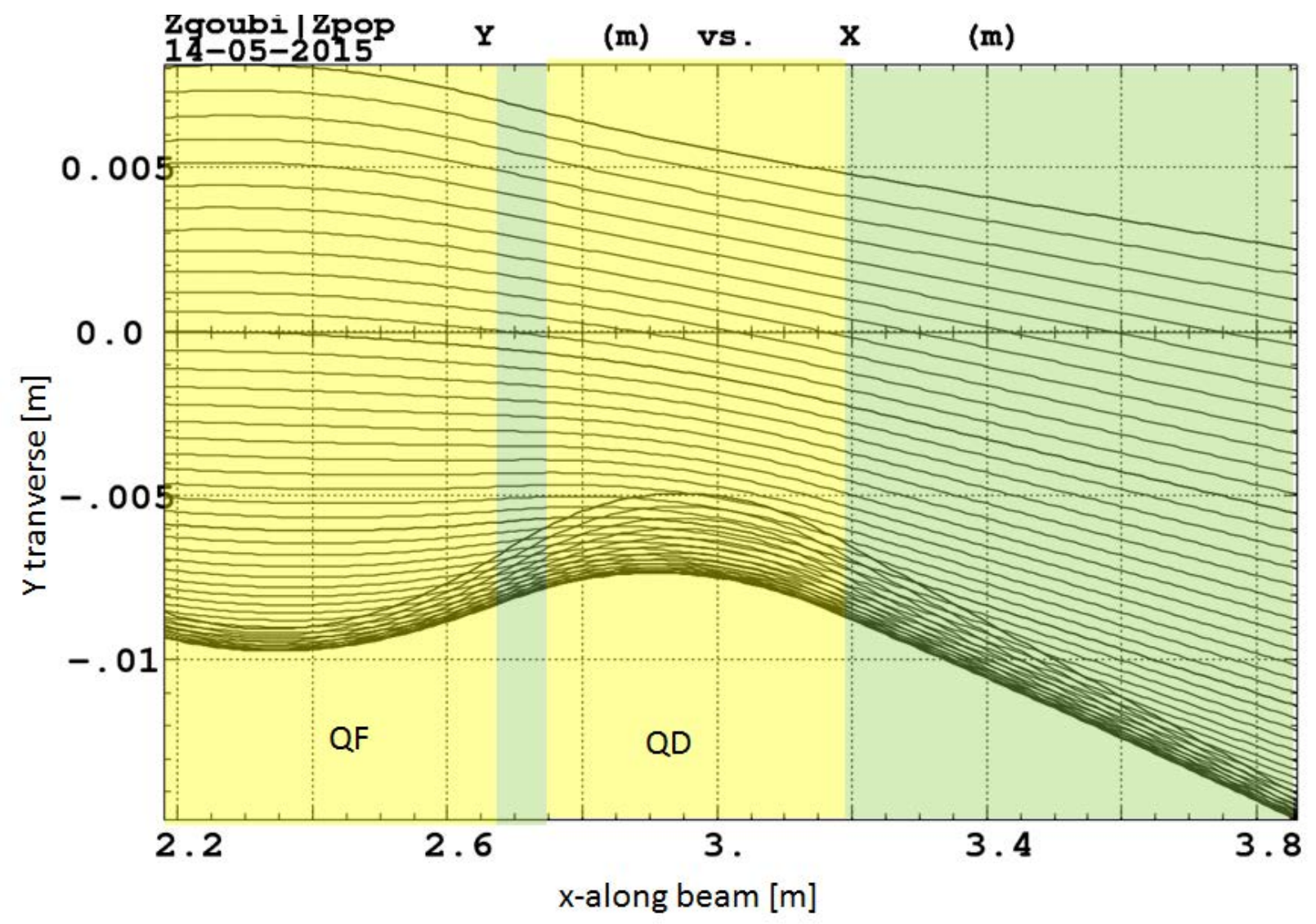

Figure 9. Closed orbit trajectories on the median plane of the cell in the energy range from 1.334 to 6.6 $\mathrm{GeV}$. This figure demonstrates the remarkable property of the FFAG concept which allows a large energy range of particles to be accommodated in a small transverse distance from -1.5 to $1.0 \mathrm{~cm}$. The coordinate system which is used by the computer code "zgoubi" is: $y$-radial, $\mathrm{x}$-along beam direction, and z-vertical.

Figure 10 shows the transverse horizontal coordinates $\left(y, y^{\prime}\right)$ at the median plane $(z=0)$ of the trajectories at the entrance of each cell as shown in Figure 9. The small crosses in Figure 10 correspond to the (y,y') coordinates of the particles at the entrance of a cell which is made of hard-edge quadrupoles. The small rectangles correspond to the $\left(\mathrm{y}, \mathrm{y}^{\prime}\right)$ coordinates at the entrance of a cell made with realistic fields as calculated by the OPERA computer code. To ascertain that the 3D field map as calculated by the OPERA code does not contain any abnormalities, we plot the $\mathrm{B}_{\mathrm{y}}$ component of the field map on Figure 11 along each particle trajectory appearing in Figure 9. Calculations of the closed orbits with cells comprised of "hard edge" magnets having the same integrated quadrupole field provide very similar results with the calculations above. This indicates that the fringe fields do not affect significantly the formation of the closed orbits. 


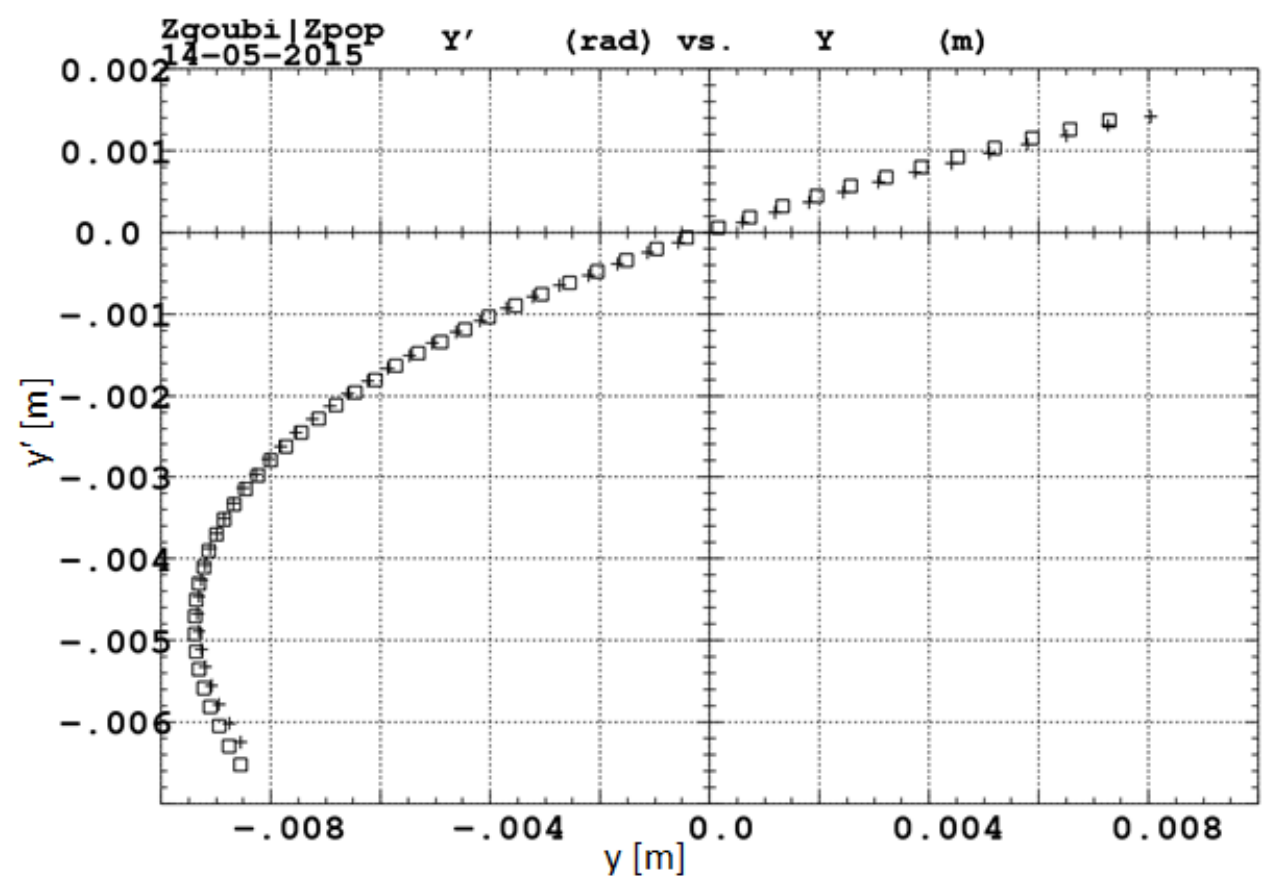

Figure 10. The transverse coordinates $\left(y, y^{\prime}\right)$ at the median plane of the closed orbit trajectories, shown in Figure 9, at the entrance of each cell. The crosses correspond to the cell made of hardedge quadrupoles, and the rectangles to a cell with fields as calculated by the OPERA computer code.

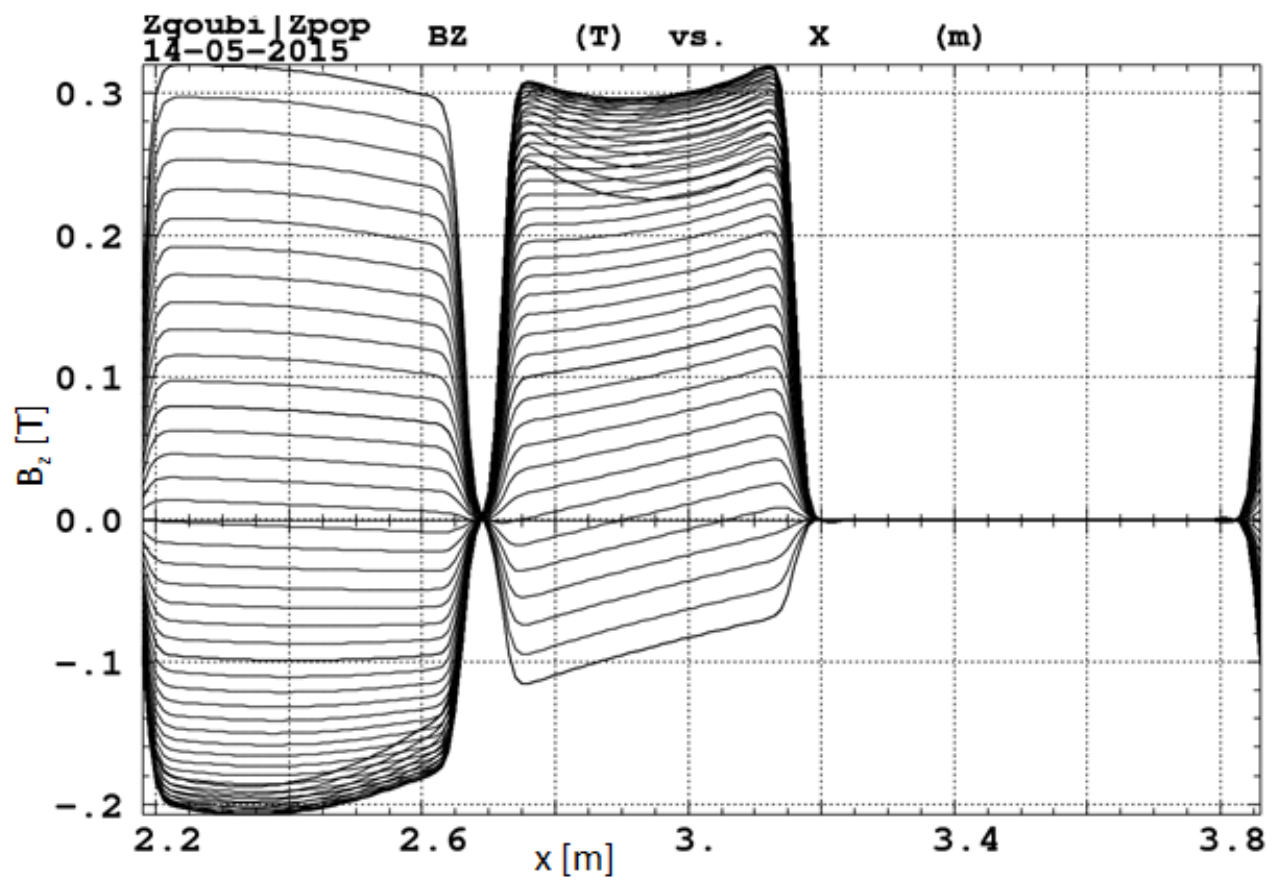

Figure 11. Plot of the vertical $\mathrm{B}_{z}$ component of the field along each particle trajectories shown in Figure 9. 
The tunes $Q_{x}, Q_{y}$ and the chromaticities $\xi_{x}$, $\xi_{y}$ per cell.

The betatron tunes provide good information on the stability of each trajectory. Likewise the chromaticities provide information of the stability of the beam bunch with particles having finite momentum spread. Figure 12 is a plot of the tunes $Q_{x}, Q_{y}$, and the chromaticities $\xi_{x}, \xi_{y}$, as calculated by the computer code "zgoubi”, as a function of the particle's energy.

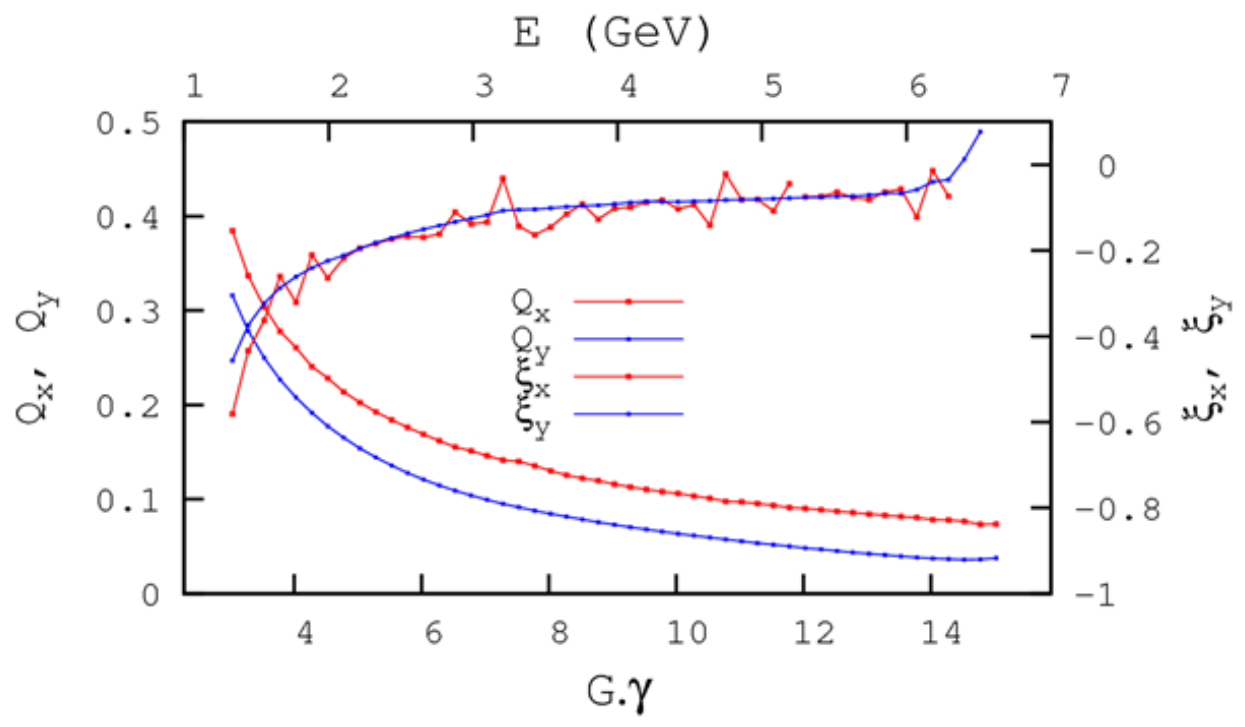

Figure 12. Plot of the tunes $\mathrm{Q}_{\mathrm{x}}, \mathrm{Q}_{\mathrm{y}}$, and the chromaticities $\xi_{\mathrm{x}}, \xi_{\mathrm{y}}$, as calculated by the computer code "zgoubi", as a function of the particle's Energy. The field map from OPERA-3D is used.

Similar calculations on the tunes $\mathrm{Q}_{\mathrm{x}}, \mathrm{Q}_{\mathrm{y}}$, and the chromaticities $\xi_{\mathrm{x}}, \xi_{\mathrm{y}}$, done with "zgoubi” with cells containing "hard edge" pure quadrupole magnets are plotted on Figure 13.

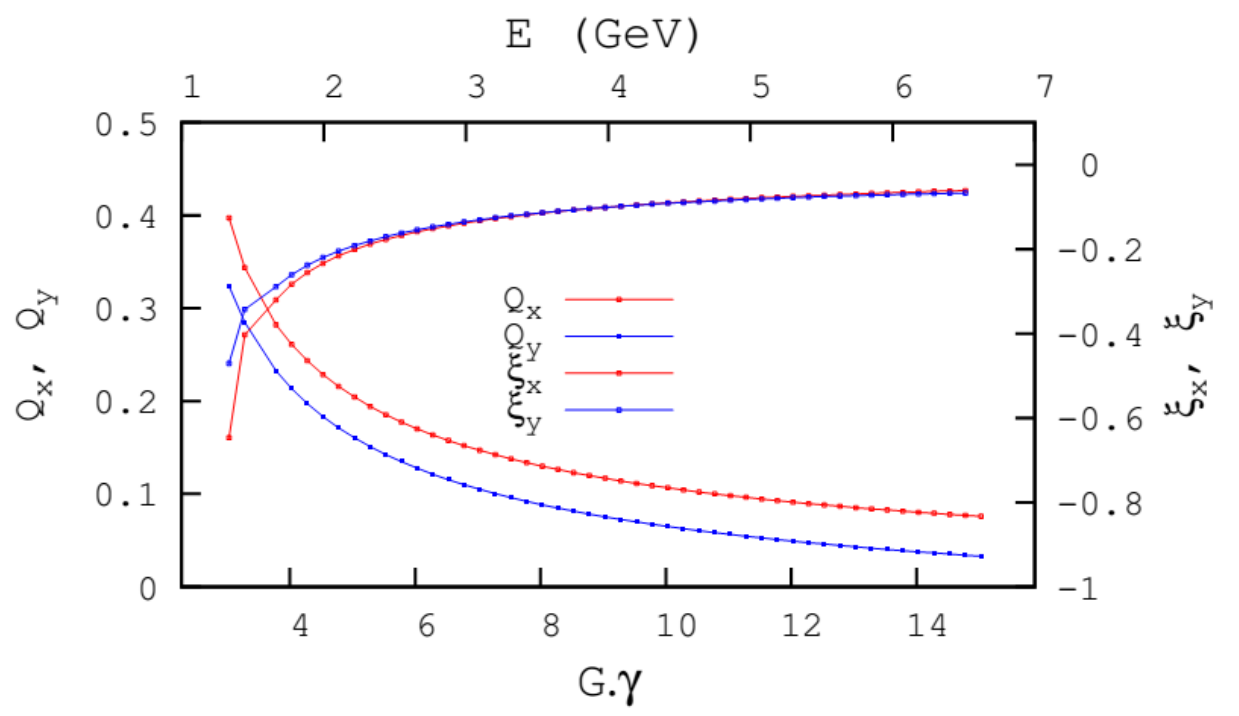

Figure 13. Plot of the tunes $\mathrm{Q}_{\mathrm{x}}, \mathrm{Q}_{\mathrm{y}}$, and the chromaticities $\xi_{x}, \xi_{y}$, as calculated by the computer code "zgoubi”, as a function of particle energy. The cells are comprised of "hard edge” pure quadrupoles. 
Maximum projected beam emittances $\varepsilon_{\mathrm{x}}$, and $\varepsilon_{\mathrm{y}}$ for each energy.

To obtain a measure of the maximum beam acceptance that each closed orbit may transport in the ring, the "zgoubi" code calculates the maximum phase space $\left(\mathrm{y}, \mathrm{y}^{\prime}\right)$ which corresponds to each closed orbit, and is transported along the 1272 cells. The vertical phase space $\left(\mathrm{z}, \mathrm{z}^{\prime}\right)$ at the entrance of the arc is assumed to have zero area. Figure 14 shows the maximum invariants $\left(y, y^{\prime}\right)$ of five ellipses which correspond to the closed orbits 1.3, 2.6, 3.9, 5.2, and $6.6 \mathrm{GeV}$

Similar calculations for maximum area $\left(\mathrm{z}, \mathrm{z}^{\prime}\right)$ of the vertical phase space appear in Figures 15 which shows the maximum invariants (z,z') for particles with energies 1.3, 2.6, 3.9, 5.2, and 6.6 GeV. The initial horizontal phase space $\left(y, y^{\prime}\right)$ is assumed to be zero.

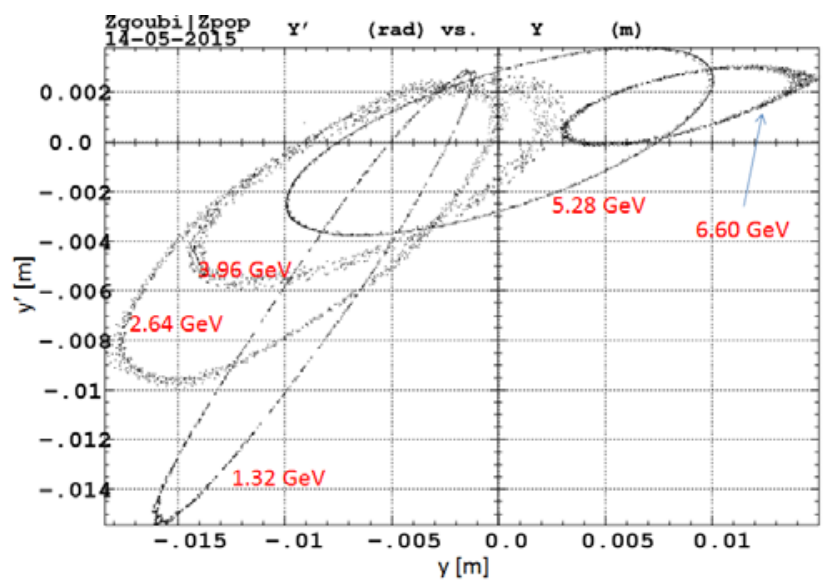

Figure 14. Maximum phase space areas (y,y') transported over 1272 cells as they appear at the exit of the last cell. Each phase space area corresponds to the closed orbits of particles with energies 1.3, 2.6, 3.9, 5.2, and $6.6 \mathrm{GeV}$.

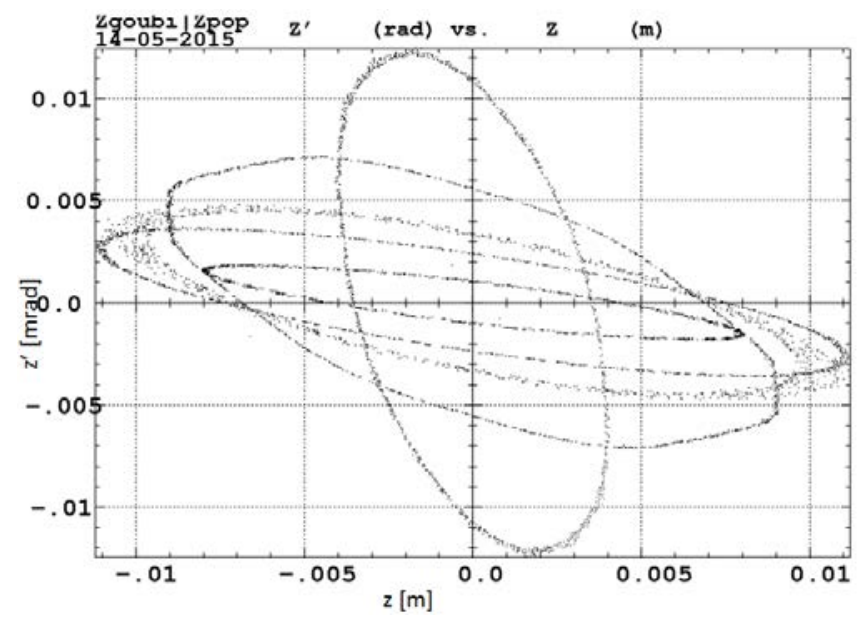

Figure 15. Maximum vertical (z,z') phase space at the exit of 1272

cells. Each phase space area corresponds to the closed orbits of particles with energies 1.3, 2.6, 3.9, 5.2, and $6.6 \mathrm{GeV}$. 
Similar calculations on a sharp cut-off model quadrupoles for five different energies show a dramatic increase of the transmitted horizontal beam emittance $\left(y, y^{\prime}\right)$ at the end of 1272 cells. The big difference in the size of the transmitted beam ellipses between the "sharp cut-off" model and the OPERA generated model is attributed to the assumption that the quadrupole field of the ideal magnets extends radially from $-\infty$ to $+\infty$. The field of the realistic magnet which is generated by the OPERA computer code extends only to distances within the aperture of the magnet and also the higher order magnetic multipoles of a realistic magnet restrict the acceptance of the ring.

The dynamic acceptance as a function of energy

In the previous section we presented the calculations on the maximum phase space ellipses that can be transported at the exit of 1272 cells. In this section we present calculations on the dynamic acceptance of 1272 cells as a function of energy. Starting with the maximum $\mathrm{x}$ and y coordinates of the phase space ellipses as we calculated in the previous section, the "zgoubi" computer code calculates the maximum $x$ and y particle coordinates at the entrance of each ring that can be transported at the exit of 1272 cells. Figure 16 is a plot of the dynamic acceptance for 5 energies in the range 1.3 to $6.6 \mathrm{GeV}$.

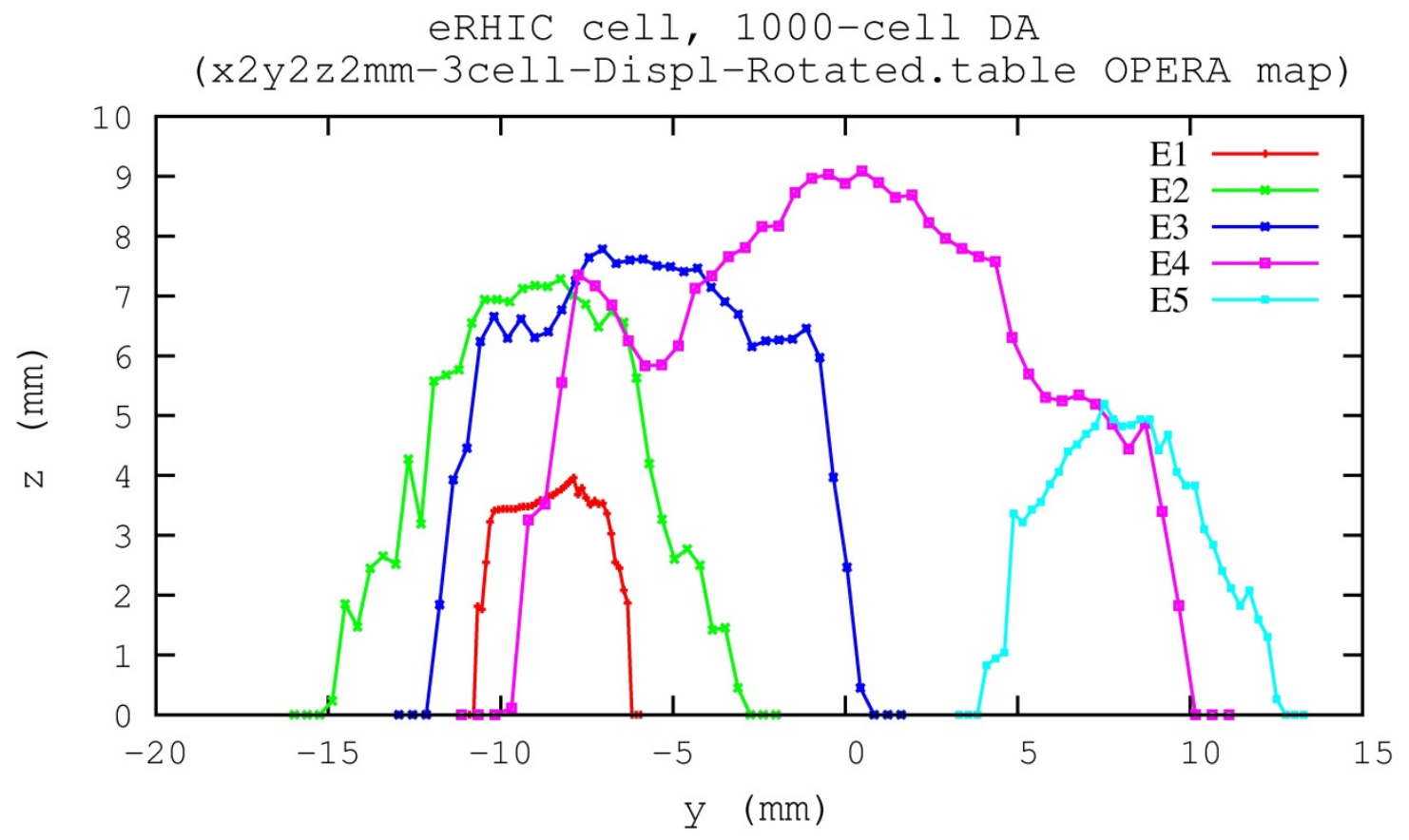

Figure 16. The dynamic acceptance of 1272 cells for 5 different energies in the range 1.3 to $6.6 \mathrm{GeV}$. The $y$-axis is the horizontal extent at the entrance of 1272 cells, of a given energy particles, as measured with respect to the closed orbit corresponding to each of the five energies. The z-axis corresponds to the vertical extent of the beam with same particular energy, at the entrance of the 1272 cells.

The y-axis in Figure 15 is the horizontal extent, of particles with one of the five energies 1.3, 2.6, 3.9, 5.2, and $6.6 \mathrm{GeV}$. The $\mathrm{y}$ and $\mathrm{z}$ coordinates are measured in reference to the closed orbit corresponding to the particular energy.

For reasons explained earlier, similar calculations of the dynamic aperture of a cell with "hard-edge" quadrupoles show a dramatic increase of the dynamic aperture. 


\section{Beta Functions}

Figure16 is a plot of the horizontal and vertical beta functions of a cell for three energies. The beta functions are calculated by the zgoubi computer code using realistic field maps. The beta functions calculated using B-fields of ideal quadrupoles are very similar to the ones plotted in Figure 16.

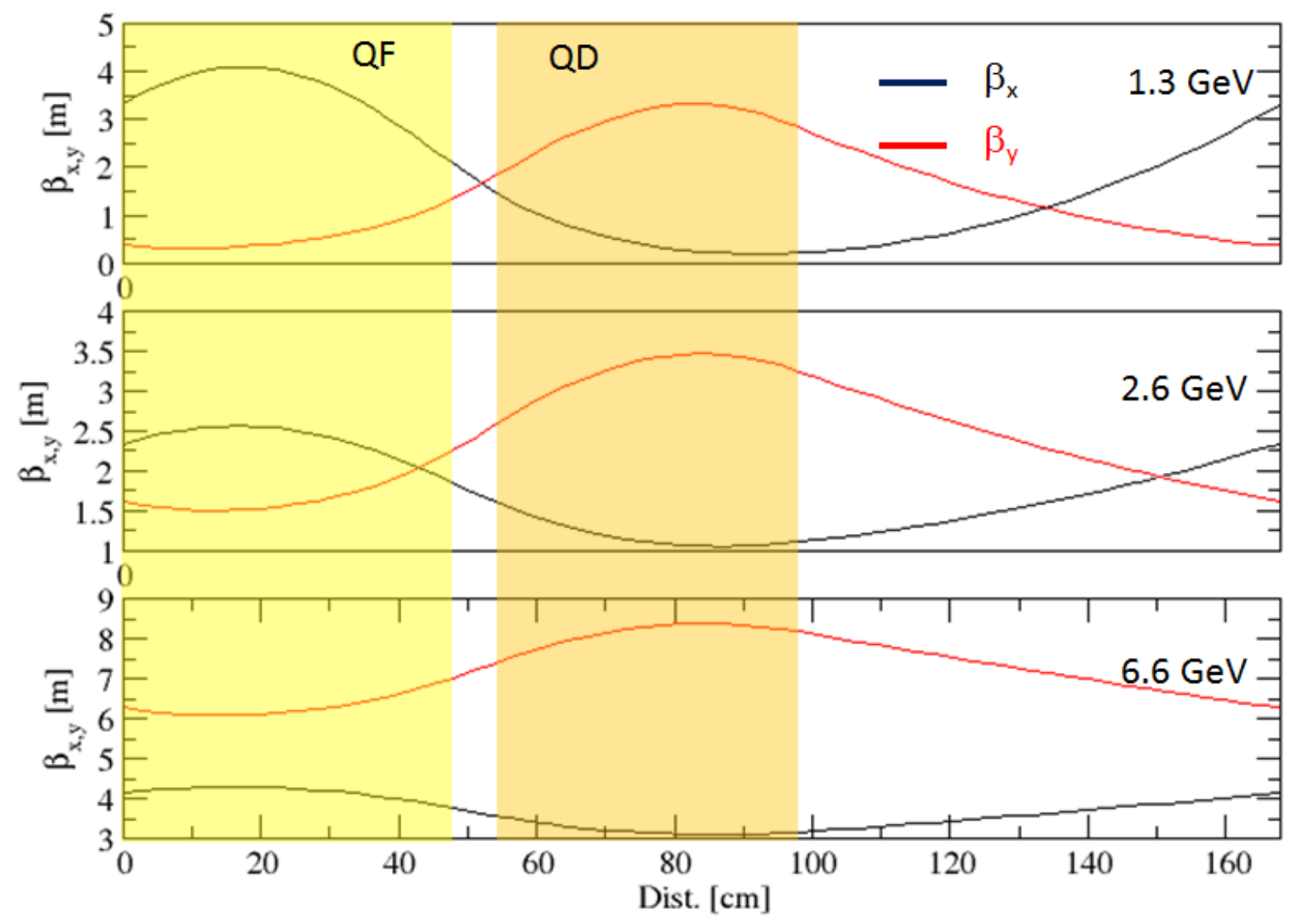

Figure 16. The horizontal and vertical beta functions for three different energies plotted along the trajectories of a cell.

\section{Conclusions}

The optical properties of a cell to be used in the FFAG lattice of the eRHIC recirculating electron ring were calculated using realistic field maps computed with the OPERA computer code. The horizontal/vertical tunes, chromaticities, indicate stability for the transported beam bunches in the required energy range. The beta functions are in good agreement with those calculated with the PTC code which uses ideal magnets for the cell. The calculated phase space for the beam acceptances and the dynamic acceptaces are large enough to accept the required beam emittances. The 3D calculations performed on a modified Halbach quadrupole are in agreement with the measurements thus these magnets can provide the desired magnetic fields in an eRHIC cell.

\section{References}

[1] arXiv:1409.1633

[2] E. Forest, E. Mcintosh and F. Schmidt, KEK Report 2002-3, CERN-SL-2002-044 (AP), 44, 3 (2002). 
[3] Vector Fields Inc.

[4] K. Halbach J. Appl. Phys. 57 (1) 15 April 1985

[5] F. Méot, Nuclear Instruments and Methods in Physics Research A 767 (2014) 112-125 